\title{
Modelling of Tunnelling Current through a Trapezoidal Potential Barrier by Using Exponential Wavefunction Approach
}

\author{
Fitriyadi $^{1, a^{*}}$ \\ ${ }^{1}$ Masters in Physics Faculty of Mathematics and Natural Sciences \\ Institut Teknologi Bandung \\ Jl. Ganesha 10 Bandung, 40132, Indonesia
}

( Received: June 08,2021, Revised: September 28,2021, Accepted: November 14,2021 )

\begin{abstract}
A tunnelling current through a trapezoidal barrier potential has been modelled. The transmittance is determined using the exponential wavefunction approach method. Furthermore, the transmittance is used to calculate the tunnelling current density by applying the Gauss-Laguerre quadrature method. The simulation results show the increasing bias voltage causes the raising tunnelling current, and an increase of temperature is proportional to the tunnelling current.
\end{abstract}

Keywords: tunnelling current, exponential wavefunction, Gauss-Laguerre quadrature

\section{INTRODUCTION}

The tunnelling effect is a quantum phenomenon studied many times because this effect gives many benefits, especially in electronic device development. The tunnelling effect is closely related to transmittance through barrier potential [1]. There are several methods to calculate the transmittance, such as transfer matrix method (TMM), airy wavefunction, WKB and exponential wavefunction.

Some researchers have simulated Tunnelling current; some of them are tunnelling current simulation through trapezoidal barrier potential using TMM [2] and the exponential wavefunction approach [3]. In 2015, Peng Zang modelled the tunnelling effect of the metalinsulator-metal (MIM) system, the benefits of the structure are for developing capacitor [5] and diode [6]. In this work, the simulation in a trapezoidal barrier potential using exponential wavefunction for MIM structure and the GaussLaguerre method is used to determine the tunnelling current density.

\section{THEORETICAL MODEL}

Fig. 1 shows the potential profile of trapezoidal barrier potential with width $Z$ and height $V_{0}$.

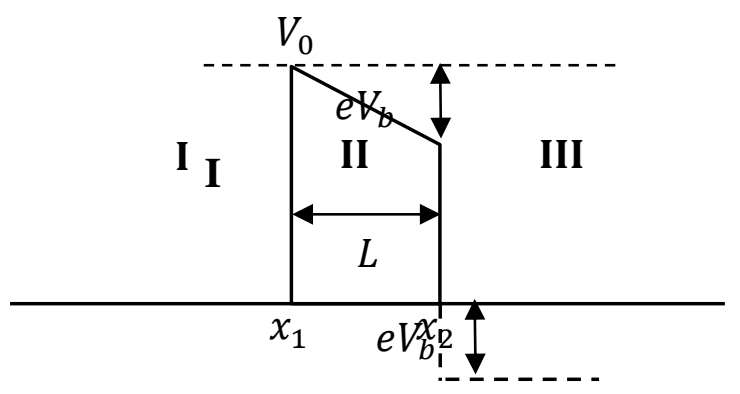

Fig. 1 Potential profile of trapezoidal barrier potential.

The potential barrier can be expressed as follows:

$$
V(x)=\quad \begin{gathered}
x<x_{1} \\
x_{1} \leq x<x_{2}
\end{gathered}
$$




$$
\left\{\begin{array}{c}
0, \\
V_{0}-\frac{e V_{b}}{x_{2}} x, \\
-e V_{b},
\end{array} \quad k_{1}=\sqrt{\frac{2 m_{1} E_{x}}{\hbar^{2}}}\right.
$$

The exponential wavefunction approach could be used to calculate the transmission coefficients through the potential barrier. The wavefunction solutions of each region are written as [3]:

$\varphi(x)=\left\{\begin{array}{ll}A e^{i k_{1} x}+B e^{-i k_{2} x}, & \text { for region I } \\ C e^{\int_{0}^{x} k_{2}\left(x^{\prime}\right) d x^{\prime}}+D e^{-\int_{0}^{x} k_{2}\left(x^{\prime}\right) d x^{\prime}}, & \text { for region II (1) } \\ E e^{i k_{3} x}, & \text { for region III }\end{array} \quad k_{3}=\sqrt{\frac{2 m_{1} E_{x}}{\hbar^{2}}\left(E_{x}+e V_{b}\right) .}\right.$

$$
k_{2}=\sqrt{\frac{2 m_{2} E_{x}}{\hbar^{2}}\left(V_{0}-\frac{e V_{b}}{L} x\right)}
$$

with,

The transmission coefficient of the system could be expressed as:

$$
\left(\frac{E}{A}\right)=2 k_{1}\left(\frac{k_{2}(L)}{k_{2}(0)}\right) e^{-i k_{3} L} \frac{\left[\left(k_{3}+\left(k_{1} \frac{k_{2}(L)}{k_{2}(0)}\right)\right) \cosh (a)\right]+i\left[\left(\frac{m_{2} k_{1} k_{3}}{m_{1} k_{2}(0)}-\frac{m_{1} k_{2}(d)}{m_{2}}\right) \sinh (a)\right]}{\left[\left(k_{3}+\left(k_{1} \frac{k_{2}(L)}{k_{2}(0)}\right)\right) \cosh (a)\right]^{2}+\left[\left(\frac{m_{2} k_{1} k_{3}}{m_{1} k_{2}(0)}-\frac{m_{1} k_{2}(d)}{m_{2}}\right) \sinh (a)\right]^{2}}
$$

with,

$$
\begin{aligned}
& a=\int_{0}^{L} k_{2} x d x \\
& a=\sqrt{\frac{2 m_{2}}{\hbar^{2}}} \frac{2 L}{3 e V_{b}}\left(\left(V_{0}-E_{x}\right)^{\frac{3}{2}}\right. \\
& \left.-\left(V_{0}-E_{x}-e V_{b}\right)^{\frac{3}{2}}\right) . \\
& J_{x}=\frac{e m k_{B} T}{2 \pi^{2} \hbar^{3}} \int_{0}^{\infty} T\left(E_{x}\right) \ln \left\{\frac{1+\exp \left[\frac{E_{F}-E_{x}}{k_{B} T}\right]}{1+\exp \left[\frac{E_{F}-E_{x}-e V_{b}}{k_{B} T}\right]}\right\} d E_{x}
\end{aligned}
$$

The tunnelling current density for this system is

$k_{B}$ is Boltzmann constant, $T$ is temperature, $\hbar$ is Planck reduced constant, and $E_{F}$ is Fermi energy [2].

The equation (8) contains infinity integral, and it could be solved using a numerical method, Gauss-Laguerre quadrature [7]:

$$
\int_{0}^{\infty} e^{-x} f(x) \approx \sum_{j=1}^{n} w_{j} f\left(x_{j}\right)
$$

with $w_{j}$ is weight, and $x_{j}$ is associated with polynomial $L_{n}(x)=\frac{e^{x} d^{n}}{d x^{n}}\left(e^{-x} x^{n}\right)$. 

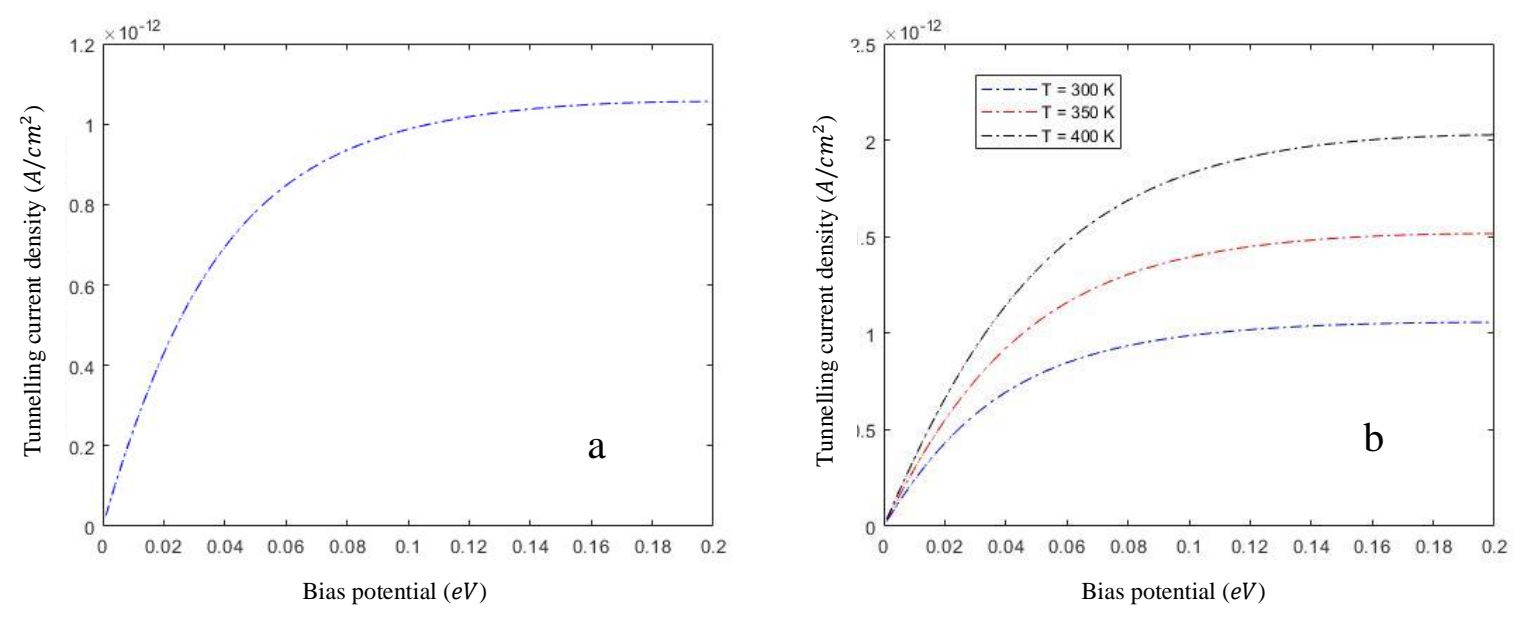

Fig. 2 (a) Tunnelling current density vs bias potential (b) Tunnelling current density in various temperature.

\section{RESULTS AND DISCUSSION}

Modelling of tunnelling current in a system with a height of the potential is 0.8 , width $5 \mathrm{~nm}$, and the Fermi energy is $0 \mathrm{eV}$ is illustrated in Fig. 2a. It shows an increase tunnelling current when bias potential $e V_{b}$ is increased. This phenomenon happened because the electron could pass the barrier quickly caused the bias voltage.

The value of tunnelling current density depends on temperature. Fig. 2(b) shows that when increased the temperature, the tunnelling current density went to a higher value in the same bias potential. It means, higher temperature makes higher tunnelling current density.

\section{CONCLUSION}

In conclusion, by the results, the bias potential proportional to tunnelling current density. Additionally, higher temperature will make higher tunnelling current density in the same bias potential value.

\section{REFERENCES}

[1] Kartiwa, G. M., Noor, F. A., \& Sulthoni, M. A. (2019). Electron Transmittance Profile Through Non-linear Potential Barrier of Sharp-shaped Electrode. IEEE.
[2] Noor, F. A., Nabila, E., Mardianti, H., Istiqomah, T. A., \& Kairurrijal. (2019). Modelling of Electron Transmittance and Tunneling Current through a Trapezoidal Potential Barrier by Considering the Spin Polarization Effect. Journal of Physics.

[3] Nabila, E., Noor, F. A., \& Khairurrijal. (2017). Comparison of Electron Transmittance and Tunneling Current through a Trapezoidal Potential Barrier with Spin Polarization Consideration by using Analytical and Numerical Approaches. IOP Conference Series: Materials Science and Engineering.

[4] Zhang, P. (2015). Scaling for Quantum Tunneling Current in Nano- and Subnanoscale Plasmonic Junctions. Scientific Reports.

[5] Tsutsumi, T., Sugitani, S., Nishimura, K., \& Ida, M. (2011). New Stacked MetalInsulator-Metal Capacitor with High Capacitance Density for Future InP-Based ICs. Japanese Journal of Applied Physics.

[6] Grover, S., \& Moddel, G. (2011). Applicability of Metal/Insulator/Metal (MIM) Diodes to Solar Rectennas. IEEE Journal of Photovoltaics.

[7] Vallèe, T. (2018). Computing the zeros and weights of Gauss-Laguerre and GaussHermite quadratures: 2 Matlab files. $H A L$. 